\title{
Boundary element formulation for the analysis of transferred potentials in electrical installations
}

\author{
I. Colominas ${ }^{1 *}$, F. Navarrina, M. Casteleiro \\ Group of Numerical Methods in Engineering, GMNI, Civil Engineering School, Universidad de La Coruña, Campus de Elviña, 15192, La Coruña, Spain
}

Received 3 October 2002; accepted 3 October 2003

Available online 12 August 2004

\begin{abstract}
In this work we present a BEM numerical formulation for the analysis of a common problem in electrical engineering practice, that is, the existence of transferred earth potentials in a grounding installation [IEEE Guide for safety in AC substation grounding (2000)]. The transfer of potentials between the grounding area to outside points by buried conductors, such as communication or signal circuits, neutral wires, pipes, rails, or metallic fences, may produce serious safety problems [Comput Methods Appl Mech Eng, 174 (1999) p. 73]. Thus, in this paper we summarize the BE numerical approach and we present a new technique for the transferred potential analysis. Finally, we show some examples by using the geometry of real grounding systems.
\end{abstract}

(C) 2004 Civil-Comp Ltd and Elsevier Ltd. All rights reserved.

Keywords: BEM numerical methods; Grounding; Transferred earth potential

\section{Introduction}

A safe grounding system has to guarantee the integrity of equipment and the continuity of the service under fault conditions (providing means to carry and dissipate electrical currents into the ground) and to safeguard that persons working or walking in the surroundings of the grounded installation are not exposed to dangerous electrical shocks. To achieve these goals, the equivalent electrical resistance of the system must be low enough to assure that fault currents dissipate mainly through the grounding grid into the earth, while maximum potential differences between close points on the earth surface must be kept under certain tolerances (step, touch and mesh voltages) [1,3].

In the last four decades, the operation of grounding systems has been extensively analyzed, and several methods for analysis and design have been proposed. Most of these methods are based on the professional experience, on semiempirical works, on experimental data obtained from scale

\footnotetext{
* Corresponding author.

E-mail address: icolominas@udc.es (I. Colominas).

${ }^{1} \mathrm{http} / / /$ caminos.udc.es/gmni
}

model assays and laboratory tests, or on intuitive ideas. Unquestionably, these contributions represented an important improvement in the grounding analysis area, although some problems have been systematically reported, such as the large computational costs required in the analysis of real cases, the unrealistic results obtained when segmentation of conductors is increased, and the uncertainty in the margin of error [1,3-5].

The electrical current dissipation into the soil is a wellknown phenomenon which equations can be stated from Maxwell's Electromagnetic Theory. Nevertheless, their application and resolution for the computing of grounding grids of large installations in practical cases present some difficulties. Evidently, no analytical solutions can be obtained for most of real problems. On the other hand, the characteristic geometry of grounding systems (a mesh of interconnected bare conductors with a relatively small ratio diameter-length) makes very difficult the use of standard numerical methods. Thus, the use of techniques commonly applied for solving boundary value problems, such as finite elements or finite differences, is extremely costly since the discretization of the domain (the ground excluding the electrode) is required. Therefore, obtaining sufficiently 
accurate results should imply unacceptable computing efforts in memory storage and CPU time.

For all these reasons, the authors have proposed in the last years a numerical approach based on the transformation of the differential equations that govern the physical phenomena onto an equivalent boundary integral equation and the subsequent application of the Boundary Element Method. Consequently, the boundary element approximations are made only on the boundary of the domain $[6,7]$. The BEM formulation proposed for the authors for the analysis of grounding systems embedded in uniform soils models $[2,8]$ can be stated in the general framework of the Symmetric Galerkin Boundary Element approaches $[9,10]$. Thus, the statement of a variational form based on a weighted-residual approach of the boundary integral equation and the selection of a Galerkin type weighting lead to a general symmetric formulation, from which it is possible to derive specific numerical algorithms of high accuracy [2]. Furthermore, the development of this BEM approach has allowed to explain from a mathematical point of view the anomalous asymptotic behaviour of the clasical methods proposed for grounding analysis, and to identify rigorously the sources of error [5]. This boundary element approach has been implemented in a Computer Aided Design system for grounding analysis [11] that allows the analysis of real earthing installations in real-time using conventional computers.

Recently, we have proposed a generalization of the boundary element formulation for grounding grids embedded in layered soils [12,13]. This is a very challenging problem with important consequences in the grounding design from the safety point of view [1]. This type of model is frequently used when there are important differences in the electrical properties of the soil. This is the case when the excavation process during the construction of the substation produces a stratified soil, or as a consequence of a chemical treatment of the soil applied in the surroundings of the earthing system to improve the performance of the grounding electrode, or due to the specific geological characteristics of the substation site, to mention some examples.

Now, we focus our attention on a common and important engineering problem in the grounding field: potential can be transferred to other grounded conductors in the vicinity of the earthing installation, and subsequently it could reach distant points through communication or signal circuits, neutral wires, pipes, rails, or metallic fences. This effect could produce serious safety problems that should be estimated somehow [1].

In this paper, we present a Boundary Element numerical formulation for the analysis of transferred potentials in grounding installations and its implementation in a Computer Aided Design system for grounding analysis. Furthermore, an application to a practical case by using the geometry of a real earthing system is presented.

\section{Mathematical model of the problem of the electrical current dissipation into a soil}

Maxwell's Electromagnetic Theory is the starting point to derive the equations that govern the dissipation of electrical currents into a soil. Thus, restricting the analysis to the electrokinetic steady-state response and neglecting the inner resistivity of the earthing conductors (potential can be assumed constant at every point of the grounding electrode surface), the $3 \mathrm{D}$ problem can be written as

$\operatorname{div}(\boldsymbol{\sigma})=0, \quad \boldsymbol{\sigma}=-\gamma \operatorname{grad}(V)$ in $E$

$\boldsymbol{\sigma}^{t} \mathbf{n}_{\mathbf{E}}=0$ in $\Gamma_{E} ;$

$V=V_{I} \quad$ in $\quad \Gamma ; \quad V \rightarrow 0, \quad$ if $\quad|\mathbf{x}| \rightarrow \infty$

where $E$ is the earth, $\gamma$ is its conductivity tensor, $\Gamma_{E}$ is the earth surface, $n_{E}$ is its normal exterior unit field and $\Gamma$ is the electrode surface [2]. Therefore, the solution to Eq. (1) gives potential $V$ and current density $\sigma$ at an arbitrary point $x$ when the electrode attains a voltage $V_{I}$ (Ground Potential Rise, or GPR) with respect to remote earth. Next, for known values of $V$ on $\Gamma_{E}$ and $\sigma$ on $\Gamma$, it is straightforward to obtain the design and safety parameters of the grounding system [2].

Depending on the type of soil model considered, it is possible to obtain different approaches. Since the techniques presented in this paper can be extended to more sophisticated soil models, such as the layered ones [12,13], and our objective is to analyze the problem of the transferred potentials in grounding systems, we will consider the simplest soil model, that is, the homogeneous and isotropic soil model [1,2]. Consequently, the conductivity tensor $\gamma$ can be substituted by an apparent scalar conductivity $\gamma$ that must be experimentally obtained [1]. Furthermore, if one takes into account that the surroundings of the substations site are levelled and regularized during its construction (then the earth surface can be assumed horizontal), the application of the 'method of images' and Green's Identity yields the following integral expression [2] for the potential $V$ at an arbitrary point $x \in E$, in terms of the unknown leakage current density $\sigma(\xi)$ at any point $\xi$ of the electrode surface $\Gamma \subset E\left(\sigma=\sigma^{t} n\right.$ being $n$ the normal exterior unit field to $\Gamma$ ):

$$
\begin{aligned}
& V(\mathbf{x})=\frac{1}{4 \pi \gamma} \iint_{\xi \in \Gamma} k(\mathbf{x}, \xi) \sigma(\xi) \mathrm{d} \Gamma, \\
& k(\mathbf{x}, \xi)=\frac{1}{|\mathbf{x}-\xi|}+\frac{1}{\left|\mathbf{x}-\xi^{\prime}\right|}
\end{aligned}
$$

where $\xi^{\prime}$ is the symmetric of $\xi$ with respect to the earth surface [2].

Now, since integral expression (2) also holds on $T$, where the potential is given by the essential boundary condition 
$\left(V(\chi)=V_{T}, \forall \chi \in \Gamma\right)$, the leakage current density $\sigma$ must satisfy a Fredholm Integral Equation of the First Kind on $\Gamma$, whose variational form is given by the integral equation

$\iint_{\chi \in \Gamma} w(\chi)\left[V_{I}-\frac{1}{4 \pi \gamma} \iint_{\xi \in \Gamma} k(\chi, \xi) \sigma(\xi) \mathrm{d} \Gamma\right] \mathrm{d} \Gamma=0$,

which must hold for all members $w(\cdot)$ of a class of functions defined on $\Gamma$ [2]. It is important to remark that obtaining the leakage current density $\sigma$ from Eq. (3) is the objective of the problem, because the potential at any point (and, of course, on the earth surface) can be straightforwardly computed by means of Eq. (2). And if the potential values are known, then the safety design parameters of the grounding system (touch, step and mesh voltages, for example) can also be immediately obtained [2].

At this point, since the unknown function $\sigma$ is defined on the boundary of the domain, it should be obvious that a numerical approach based on the Boundary Element Method [7] seems to be the right choice to solve integral Eq. (3) [2].

\section{Numerical approach based on the BEM}

The starting point in the development of the numerical model for solving the integral Eq. (3) is the discretization of the leakage current density $\sigma$ and of the electrode surface $T$, for given sets of $N$ trial functions $\left\{N_{i}(\xi)\right\}$ defined on $\Gamma$, and $M$ boundary elements $\left\{\Gamma^{\alpha}\right\}$ :

$\sigma(\xi) \approx \sigma^{h}(\xi)=\sum_{i=1}^{N} N_{i}(\xi) \sigma_{i}^{h}, \quad \Gamma=\bigcup_{\alpha=1}^{M} \Gamma^{\alpha}$.

Now, expression (2) for potential $V(x)$ can also be discretized as

$V(\mathbf{x})=\sum_{i=1}^{N} \sigma_{i}^{h} V_{i}(\mathbf{x}), \quad V_{i}(\mathbf{x})=\sum_{\alpha=1}^{M} V_{i}^{\alpha}(\mathbf{x})$,

where $V_{i}^{\alpha}(x)$ depends on the integral on $\Gamma^{\alpha}$ of the integral kernel $k(x, \xi)$ (given in Eq. (2)) times the trial function $N_{i}(\xi)$ [2].

On the other hand, for a given set of $N$ test functions $\left\{w_{j}(\chi)\right\}$ defined on $\Gamma$, the variational form Eq. (3) can be written in terms of the following linear system of equations, as it is usual in boundary element and finite element methods:

$\sum_{i=1}^{N} R_{j i} \sigma_{i}^{h}=v_{j} \quad j=1, \ldots, N$

being

$R_{j i}=\sum_{\beta=1}^{M} \sum_{\alpha=1}^{M} R_{j i}^{\beta \alpha} ; \quad v_{j}=\sum_{\beta=1}^{M} v_{j}^{\beta}$ where $R_{j i}^{\beta \alpha}$ depends on the integrals on $\Gamma^{\alpha}$ and on $\Gamma^{\beta}$ of the integral kernel $k(\chi, \xi)$ (given in Eq. (2)) times the trial function $N_{i}(\xi)$ and times the test function $w_{j}(\chi)$, and $v_{j}^{\beta}$ depends on the integrals on $\Gamma^{\beta}$ of the test function $w_{j}(\chi)$ [2].

As we can observe, the solution of system Eq. (6) provides the values of the unknowns $\sigma_{i}^{h}(i=1, \ldots, N)$ that are necessary to compute the potential $V$ at any point $x$ by means of Eq. (5). Besides, the other safety parameters can be easily obtained from the potential distribution and the leakage current density $\sigma$ [2].

In the present work, we focus our attention on the analysis of the transferred earth potentials in grounding systems. The starting point for this study is the numerical approach based on the BEM whose main highlights have been presented above. In the references [2,13], it can be found the whole development of the numerical formulation based on the BEM for uniform and layered soil models, including the derivation of a 1D approximated numerical approach (taking into account the real geometry of grounding systems in practical cases), and the highly efficient analytical integration techniques developed by the authors for computing terms $V_{i}^{\alpha}(\mathbf{x})$ of Eq. (5) and $R_{j i}^{\beta \alpha}$ of Eq. (7) which are finally computed by means of explicit formulae. Moreover, in [2,5] a fully explicit discussion about the main numerical aspects of the BEM numerical approaches (such as the asymptotic convergence, the overall computational efficiency, and the complete explanation of the sources of error of the widespread intuitive methods) can be found.

The result is a numerical approach mathematically and numerically well-founded, and highly efficient from a computational point of view, which has been finally implemented in a Computer Aided Design system for grounding analysis in uniform and layered soil models [2,5, $8,11-14]$.

\section{The problem of transferred earth potentials}

Transferred earth potentials refer to the phenomenon of the earth potential of one location appearing at another location where there is a contrasting earth potential [15]. Specifically, during a fault condition the grounding grid of an electrical substation attains a voltage (the Ground Potential Rise, or GPR) which can be of the order of thousands of volts. This voltage (or a fraction of it) may be transferred out to a non-fault site by a ground conductor (such as metal pipes, rails, metallic fences, etc.) leaving the substation area.

This situation may produce serious hazards and must to be avoided to ensure the protection of the personnel, of the equipment and, in general, of the living beings at the nonfaulted end [16].

The importance of the problem results from the very high difference of potential finally produced, which is often possible. Main danger is of the 'touch type', i.e. when 
a person standing at a remote location far away from the substation site touches a conductor connected to the grounding grid, or touches a conductor not directly connected to the grounding grid but with a high voltage level (a fraction of the GPR) produced by an induced potential on it.

Fortunately, in most instances the potential difference will be too low to cause a shock hazard to persons or livestock. However, the difference of voltage between close points on the earth surface may be enough to produce some discomforts to sensitive persons (like children), or to affect the livestock (i.e. problems with the milk production of the cattle have been reported [17]). On the other hand, the presence of these transferred potentials due to buried conductors may also produce the anomalous operation of some electrical equipment or the distortion in the measurement instruments or electronic devices [16,18].

In references $[1,16]$ a discussion on the means that can be taken to protect against the danger of transferred earth potentials in communications circuits, rails, low-voltage neutral wires, portable equipment and tools supplied from substation, piping, auxiliary building and fences can be found.

Generally, there are two main cases of transferred potentials: (a) the transference of the Ground Potential Rise to distant points of the grounding site by means of a conductor directly linked to the earthing system; and (b), the transference of a fraction of the Ground Potential Rise to distant points of the grounding site by the existence of conductors close to the earthing grid but not directly connected to it (these conductors are energized to a fraction of the GPR when an eddy current is derived to the grounding grid during a fault condition). In both cases, the potential distribution on the earth surface will be significantly modified. This could imply a serious safety problem when it affects non-protected areas [15].

Obviously, the best way to deal with these problems is to avoid transferred potentials. However, this is not always possible. For example, in large electrical substations it is often routed a railway spur to facilitate the installation of high-power transformers or other large equipment. These railroad tracks frequently extend beyond the substation site, and they can transfer dangerous potentials during a fault condition in the grounding system [19].

The practices generally used to prevent these hazardous voltages (e.g. the use of isolation joints or the removal of several rail sections) are based on the combination of a good engineering expertise, some very crude calculations and, in a few cases, field measurements $[1,16,18-20]$.

Now, with the development of new computer methods for grounding analysis, a more accurate determination of the dangerous transferred earth potentials can be performed.

In the next section, we present a numerical approach based on the Boundary Element Method for the analysis of transferred earth potentials in grounding systems.
The starting point of this approach will be the BEM formulation presented in the previous section.

\section{Analysis of transferred earth potentials}

The analysis of transferred earth potentials when the extra-conductors and the grounding grid are both electrically connected does not imply a significant change in the numerical approach. As it has been previously discussed, since the inner resistivity of all conductors is neglected, the potential can be assumed constant at every point of their surfaces. Consequently, during fault conditions all conductors are energized to the Ground Potential Rise and the extra-conductors also work as 'grounded electrodes', leaking electrical current into the ground. So, the extraconductors are formally part of the grounding grid, and they must be included in the earthing analysis as part of the grid [14].

The problem of transferred potentials if there is no a direct electrical connection between the extra-conductors and the grounding grid is more difficult to deal with. The main problem is that the extra-conductors attain an unknown voltage (i.e. a fraction of the GPR) due to their closeness to the grounding grid when a fault condition occurs. Our objective is to obtain this voltage, and the rest of safety parameters of the grounding system (potential distribution on the earth surface, the step and touch voltages, the equivalent resistance, etc.).

In the following explanation, we call 'active grid' the set of electrodes which form the grounding grid (energized to the GPR), and 'passive grid' the extra-conductors (which attain a fraction of the GPR) not connected to the earthing grid. It is obvious that the importance of these transferred potentials will decrease if the 'passive grid' is far from the 'active grid', and their effects will be local; however it may produce non-negligible differences of potential on the earth surface in unexpected areas, even outside of the substation site.

The analysis of transferred potentials from an 'active grid' to a 'passive grid' can be performed by means of a superposition of elementary states given the linear condition of the state equations. We consider two elementary states: state (1) the 'active grid' energized to $1 \mathrm{~V}$ and the 'passive grid' to $0 \mathrm{~V}$; and state (2) the 'active grid' energized to $0 \mathrm{~V}$ and the 'passive grid' to $1 \mathrm{~V}$. With these values of unitary Ground Potential Rise, we can apply the BEM numerical approach presented in Section 3 to each elementary state, and to compute the total electrical current by unit of voltage which flows from each grid: $i_{\mathrm{A} 1}, i_{\mathrm{A} 2}, i_{\mathrm{P} 1}$ and $i_{\mathrm{P} 2}$ ('A' denotes the 'active grid', 'P' the 'passive grid', and the numbers refer to each elementary state).

The final state is as follows: the 'active grid' energized to the GPR, and the 'passive grid' energized to a constant unknown potential (that is, a fraction $\lambda$ of the GPR). 
Consequently, this final state can be obtained by superposition of the previous elementary states: the state Eq. (1) weighted by the GPR of the 'active grid' $\left(V_{T}\right)$; and the state Eq. (2) weighted by a fraction of the GPR $\left(\lambda V_{\Gamma}\right)$. Finally, coefficient $\lambda$ and the total current leaked to the soil $\left(I_{\mathrm{G}}\right)$ are computed by imposing that the fault condition is produced only in the 'active grid' [14], that is by solving the linear system of equations,

$I_{\mathrm{G}}=V_{\Gamma} i_{\mathrm{A} 1}+\lambda V_{\Gamma} i_{\mathrm{A} 2} \quad 0=V_{\Gamma} i_{\mathrm{P} 1}+\lambda V_{\Gamma} i_{\mathrm{P} 2}$.
Once the fraction $\lambda$ of the GPR is known in the 'passive grid', it is possible to compute the potential distribution on the earth surface, and consequently, to calculate the touch and step voltages in all points of the substation site and in its surroundings.

\section{Example of transferred potential analysis}

The above methodology has been applied to the analysis of the transferred earth potentials by railway
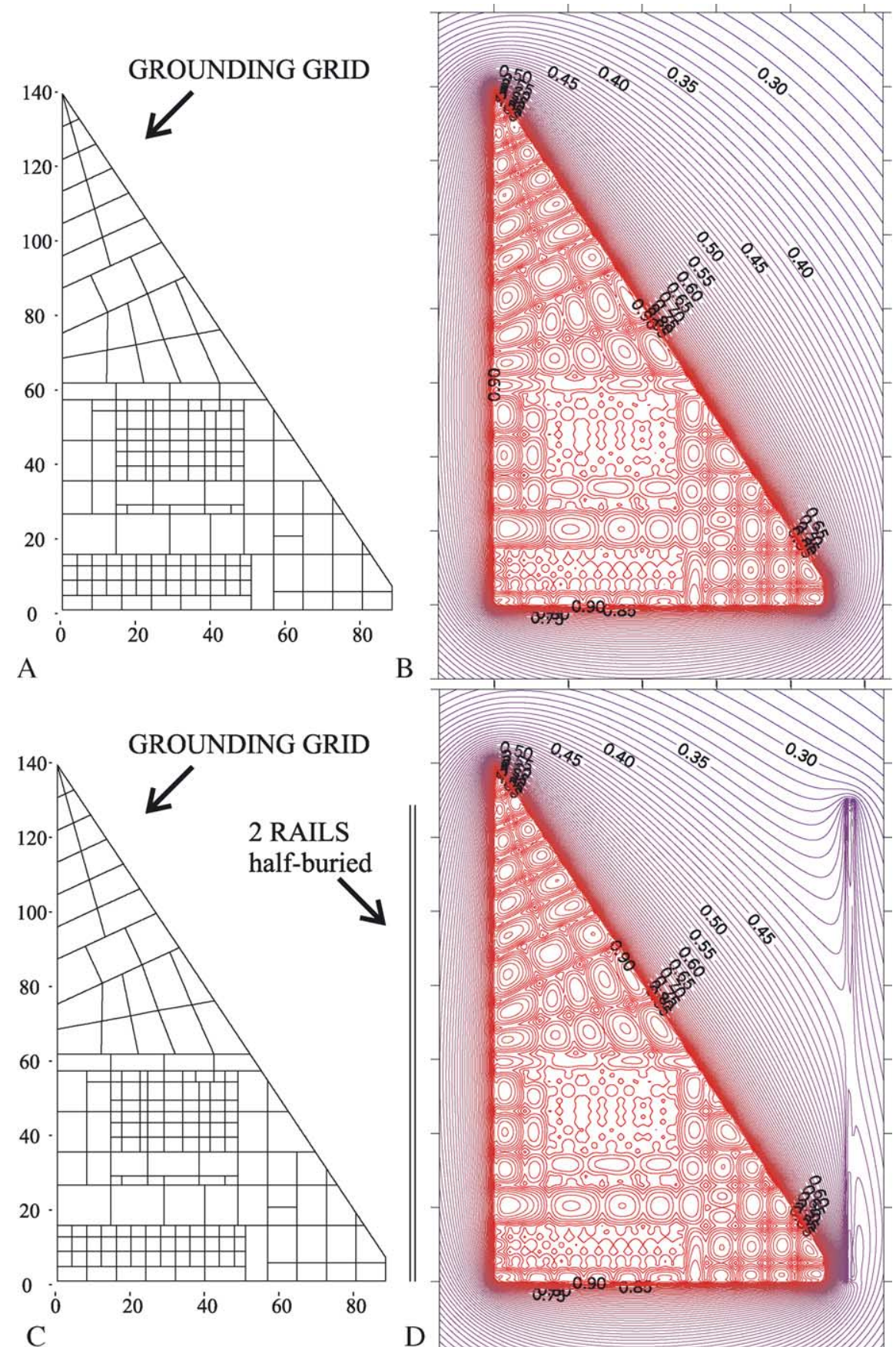

B

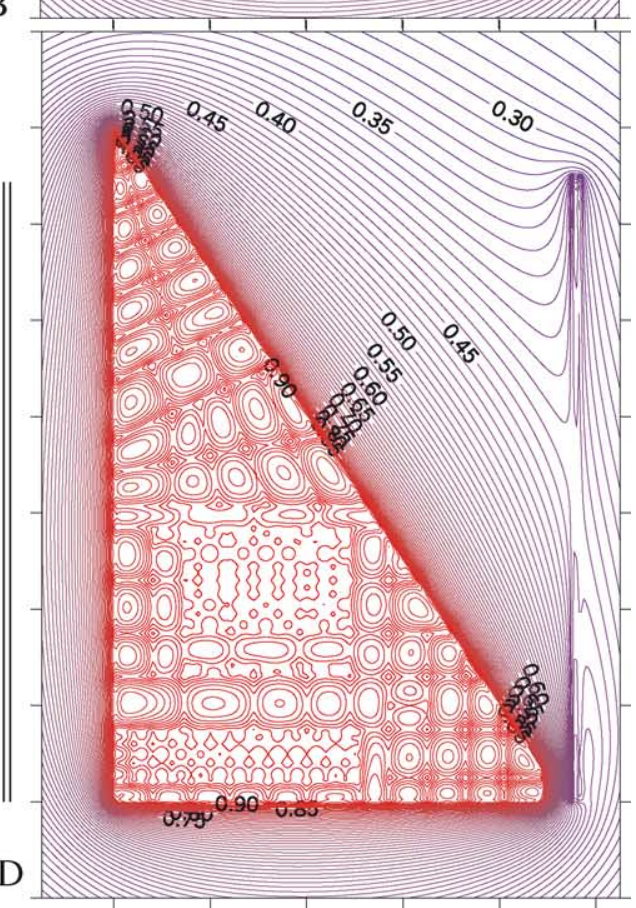

Fig. 1. (A) Plan of the grounding grid; (B) Potential distribution $(\times 10 \mathrm{kV})$ on the earth surface; (C) Plan of the grounding grid and situation of the railway tracks; (D) Potential distribution $(\times 10 \mathrm{kV})$ considering the transferred potentials by the tracks. 
Table 1

Grounding system: data and BEM numerical model

\begin{tabular}{ll}
\hline Data & \\
\hline Number of electrodes & 408 \\
Diameter of electrodes & $12.85 \mathrm{~mm}$ \\
Depth of the grid & $800 \mathrm{~mm}$ \\
Max. dimensions of grid & $145 \times 90 \mathrm{~m}^{2}$ \\
Soil resistivity & $60 \Omega \mathrm{m}$ \\
GPR & $10 \mathrm{kV}$ \\
BEM numerical model & \\
Type of approach & Galerkin \\
Type of 1D element & Linear \\
Number of elements & 408 \\
Degrees of freedom & 408 \\
\hline
\end{tabular}

tracks close to the grounding system of an electrical substation. In order to show the feasibility of this approach in a practical case, we have chosen the geometry of a real grounding grid, whose plan is shown in Fig. 1(A). The earthing grid is formed by 408 cylindrical conductors (whose diameter is $12.85 \mathrm{~mm}$ ) and buried to a depth of $80 \mathrm{~cm}$. In this study, we have considered the soil homogeneous and isotropic with an apparent scalar resistivity of $60 \Omega \mathrm{m}$. The GPR is $10 \mathrm{kV}$.

Table 1 summarizes the general data of the grounding system and the BEM numerical model. Fig. 1(B) shows the potential distribution on the earth surface when the grounding grid is energized to the GPR.

Next, we have analyzed the same grounding system but now taking into account the existence of two railway tracks in the vicinity of the substation site, as it is shown in Fig. 1(C). As it was previously exposed, this is a common situation in electrical substations and generating plants where a railway spur is used for the installation of large equipment, the fuel supplying, etc. [19].

Table 2 summarizes the geometrical characteristics of the tracks. Both systems (the grounding grid of the substation and the tracks) are not directly connected. Therefore, when the earthing grid of the substation is energized to the GPR $(10 \mathrm{kV})$ during a fault condition (that is, it is the 'active grid'), the tracks are energized to a fraction of this GPR (i.e. the tracks are a "passive grid') producing the transference of potentials in their vicinity.

The analysis of transferred earth potentials has been performed by using the proposed BEM approach and the superposition of elementary states presented previously. The fraction of the GPR of the 'passive grid' turns out to be of $\lambda=0.448$. Fig. 1(D) shows the potential distribution on the earth surface.

As expected, it is obvious that there are insignificant differences in the potential distribution on the earth surface in the area covered by the grounding grid of the substation, neither in the touch and step voltages, nor in the equivalent
Table 2

Railway tracks: characteristics

\begin{tabular}{ll}
\hline Data & \\
\hline Number of tracks & 2 \\
Length of the tracks & $130 \mathrm{~m}$ \\
Distance between the tracks & $1668 \mathrm{~mm}$ \\
Diameter of the tracks & $94 \mathrm{~mm}$ \\
Depth & $100 \mathrm{~mm}$ \\
\hline
\end{tabular}

resistance of the grounding system ( 0.336 versus $0.312 \Omega$ if the rail tracks are considered).

However, important differences in the potential distribution on the earth surface in the surroundings of the railway tracks can be noted. The comparison between Fig. 1(B) and (D) shows that in some areas close to the rail tracks, important potential gradients are produced. The danger is not the magnitude of the transferred potentials, but the difference of potential values: in some points in the vicinity of the tracks, we compute step voltages $(0.104 \mathrm{kV})$ ten times higher than the step voltages computed without considering the transferred potentials by the tracks. Furthermore, as it can be observed, these potential gradients are produced in distant points, outside the electrical substation, and surely in a non-protected area.

\section{Conclusions}

In this paper, we have revised the mathematical model of the physical phenomenon of the electrical current dissipation into the soil through a grounding grid. We have summarized the main highlights of the numerical approach based on the BEM proposed by the authors for grounding analysis in uniform soil models.

Furthermore for the first time a numerical approach for the computational analysis of transferred earth potentials by electrical conductors buried in the surroundings of a grounding system has been presented.

The numerical formulation has been implemented in a Computer Aided Design system for earthing analysis, which allows the design of grounding grids in real-time taking into account the effects of the transference of potential to distant points of the substation site.

Nowadays, we are working on the generalization of the transferred earth potential analysis to non-uniform soil models.

\section{Acknowledgements}

This work has been partially supported by the 'Ministerio de Ciencia y Tecnología (project \#DPI20010556)' of the Spanish Government cofinanced with FEDER funds. 


\section{References}

[1] IEEE Std.80, IEEE Guide for safety in AC substation grounding. New York; 2000.

[2] Colominas I, Navarrina F, Casteleiro M. A boundary element numerical approach for grounding grid computation. Comput Methods Appl Mech Eng 1999;174:73-90.

[3] Sverak JG. Progress in step and touch voltage equations of ANSI/IEEE Std.80. IEEE Trans Power Deliv 1999;13: 762-7.

[4] Garrett DL, Pruitt JG. Problems encountered with the APM of analyzing substation grounding systems. IEEE Trans Power Apparatus Syst 1985;104:3586-96.

[5] Navarrina F, Colominas I, Casteleiro M. Why do computer methods for grounding produce anomalous results? (in press) IEEE Trans Power Deliv 2003;18:1192-202.

[6] Brebbia CA, Telles JCF, Wrobel LC. Boundary element techniques. UK: Verlag; 1984.

[7] Banerjee PK. The boundary element method in engineering. London: Mc Graw-Hill; 1995.

[8] Navarrina F, Colominas I, Casteleiro M. Analytical integration techniques for earthing grid computation by BEM. In: Numerica methods in engineering and applied sciences. Barcelona: CIMNE; 1992, p. 1197-206.

[9] Johnson C. Numerical solution of partial differential equations by the Finite Element Method. Cambridge, USA: Cambridge University Press; 1987.

[10] Bonnet M, Maier G, Polizzotto C. On the symmetric Galerkin boundary element method. Appl Mech Rev 1998;51:669-704.
[11] Casteleiro M, Hernández LA, Colominas I, Navarrina F. Memory and user guide of system TOTBEM for CAD of grounding grids in electrical installations, (in spanish). Civil Engineering School, Universidad de La Coruña; 1994.

[12] Colominas I, Gómez-Calviño J, Navarrina F, Casteleiro M. Computer analysis of earthing systems in horizontally and vertically layered soils. Electric Power Syst Res 2001;59:149-56.

[13] Colominas I, Navarrina F, Casteleiro M. A numerical formulation for grounding analysis in stratified soils. IEEE Trans Power Deliv 2002; 17:587-95.

[14] Colominas I. A CAD system of grounding grids for electrical installations: a numerical approach based on the Boundary Element integral method, (in spanish), Ph.D. Thesis, Civil Engineering School, Universidad de La Coruña; 1995.

[15] Shipp DD, Nichols N. Designing to avoid hazardous transferred earth potentials. IEEE Trans Ind Appl 1982;1A-A18(4).

[16] IEEE Std. 142-1991, IEEE Recommended practice for grounding of industrial and commercial power systems. New York: 1996.

[17] Dick WK, Winter DF. Computation, measurement and mitigation of neutral-to-earth potentials on electrical distribution systems. IEEE Trans Power Deliv 1987;2(2).

[18] IEEE Std. 81-1983, IEEE Guide for measuring earth resistivity, ground impedance and earth surface potentials of a ground system. New York; 1983.

[19] Garrett DL, Wallace KA. A critical analysis of grounding practices for railroad tracks in electric utility stations. IEEE Trans Power Deliv 1993;8:90-6.

[20] IEEE Std. 665-1987, IEEE Guide for generating station grounding. New York; 1987. 